\title{
The Mathematical Work with the Derivative of a Function: Teachers' Practices with the Idea of "Generic"
}

\author{
El Trabajo Matemático relativo a la Derivada de una Función: las Prácticas \\ Profesionales bajo la Idea de lo "Genérico"
}

\author{
Monica Panero* \\ Ferdinando Arzarello** \\ Cristina Sabena ${ }^{* *}$
}

\begin{abstract}
This paper investigates the introduction of the derivative notion and, specifically, the introduction of the derivative function, as a significant moment in the development of mathematical work on functions. In particular, we analyse the process of genericization that two Italian teachers conducted with their grade 13 students, in order to make them shift from the derivative at a specific point $x_{0}$ to the derivative as a global function in the $x$ variable. Specifically, we analyse the role of the teacher in the semiotic genesis of this process and investigate the role of semiotic resources therein. As a result, we highlight the importance of conducting carefully this shift from the pointwise $x_{0}$ sign to the global $x$ sign, in order to gain an actual shift in the perceived properties of the derivative function, which depends on the $x$ sign as a variable. In conclusion, we connect our findings to the model of the Mathematical Working Space of functions, with particular regard to the "visualisation" process and the semiotic axis.
\end{abstract}

Keywords: Derivative Function. Generic. Perspectives on Functions. Calculus.

\begin{abstract}
Resumen
En este artículo se estudia la introducción de la noción de derivada, especialmente de la función derivada, como un momento significativo en el desarrollo del trabajo matemático relativo a las funciones. Particularmente, se analiza el procesos de genericización que dos profesores italianos conducen con sus estudiantes de último año de liceo, con el objetivo de de hacerlos pasar de la derivada en un punto específico $x_{0}$ a la derivada como una función global de la variable $x$. Específicamente, se analiza el rol del profesor en le génesis semiótica del proceso y se investiga el rol de los recursos semióticos implicados. Como resultado, se resalta la importancia de conducir cuidadosamente el paso desde el símbolo puntual $x_{0}$ al símbolo global $x$, para ganar un cambio real en las propiedades percibidas de la función derivada, que depende del símbolo $x$ como variable. En conclusión, conectamos los resultados al modelo del espacio de trabajo matemático de funciones, con un particular énfasis en el proceso de "visualización" y el eje semiótico.
\end{abstract}

Palabras claves: Función Derivada. Generico. Perspectivas en funciones. Cálculo.

\footnotetext{
* PhD in Mathematics, Dipartimento di Matematica "G. Peano", Università di Torino. Institut Français de l’Éducation (IFÉ), ENS de Lyon, 15 parvis René Descartes - BP 700069342 Lyon Cedex 07 - France, monica.panero@ens-lyon.fr

** Full Professor of Mathematics, Dipartimento di Matematica "G. Peano", Università di Torino, Via Carlo Alberto 10 - 10123 Torino - Italy, ferdinando.arzarello@unito.it

*** Associate Professor in Mathematics Education, Dipartimento di Filosofia e Scienze dell'Educazione, Università di Torino, Via Gaudenzio Ferrari 9/11 - 10124 Torino - Italy, cristina.sabena@ unito.it
} 


\section{Introduction}

The derivative is one of the crucial notions related to functions in secondary teaching. Some rooted algebraic practices intervene in the development of practices that are proper to Calculus, such as the use of limit computation. Hence, the introduction of the derivative is a delicate moment for both students and teachers. The Italian curriculum for the upper secondary school, in particular for the scientific section, proposes successive steps for studying functions. Students first learn to determine the domain, to analyse the sign and to detect possible intersections with Cartesian axis, as well as possible symmetries and asymptotical behaviour. Afterwards, the derivative at a point $x_{0}$ is defined as a tool for studying the variations of a function. Drawing on the work done with limits calculation, the introduction of the derivative enriches the local study of a function in a neighbourhood of a point. Subsequently, the derivative function is introduced as the derivative at "the generic point $x$ " and can be studied in its turn. In spite of the simplicity with which the definition is given at school, conceptualizing the derivative of a function as a mathematical object, and in particular as a function itself, in order to employ it as a tool (DOUADY, 1986), may not turn out to be so immediate. Indeed, students' conceptions about functions are involved, and in particular their definition of the concept of function (VINNER, 1983; SIERPINSKA, 1992; ELIA et al., 2007). Some studies underline that the transition from considering a function at a specific point to considering it on an interval is non-trivial for students (MONK, 1994). Drawing on these results, Park $(2013,2015)$ has recently studied the students' and the instructors' discourse on the derivative at a post-secondary level. She points out that the transition from the view at a specific point to the view on an interval is mainly based on symbolic notations with little explanation of how the value of the derivative changes. Students show "a mixed notion of the derivative as a function on an interval and as a point-specific object simultaneously, not fully appreciating their relation" (PARK, 2013, p. 637).

The mathematical work on functions develops thanks to the so-called games of frames (DOUADY, 1986). Typically, the students have to study algebraically some properties of a function (e.g., domain, sign) and then to interpret graphically the obtained results. This shift also implies the ability of coordinating different registers of representation (DUVAL, 1993, 2006). Another important competence to develop is the flexibility of adopting different perspectives on a function (VANDEBROUCK, 2011), in order to grasp properties that are 
valid at a specific point (i.e., pointwise properties), in an interval as well as in the whole domain (i.e., global properties), or in a neighbourhood of a point (i.e., local properties).

Drawing on the analysis carried out in Panero et al. (2015), this paper investigates the process through which the derivative function is introduced starting from the definition of the derivative at a specific point $x_{0}$. It consists in generating the generic case and it is implemented through the semiotic technique of substituting the sign $x_{0}$ with the generic $x$. For this reason, we call this process genericization and we consider it as one of the cognitive processes of the Mathematical Working Space (MWS in the following) of functions. Our objective is analysing the role of the teacher in managing the semiotic genesis of this process.

To do that, we elaborate the idea of generic in mathematics and the way it is used (§2). We will see that in order to properly enter into the genericization process we need two theoretical tools: the model of the MWS of Kuzniak and Richard (2014), and the semiotic tools as described by the semiotic bundle of Arzarello (2006) (§3). Successively ( $§ 4-6)$, we use such theoretical tools to analyse the case studies of two Italian teachers working with the derivative notion with their grade 13 students. We focus on the moment when they shift from the derivative at a specific point $x_{0}$ to the derivative as a global function in the $x$ variable, exploiting the idea of considering a generic $x$. The final sections ( $\S$ 7-8) discuss how the theoretical framework is useful for reflecting on the genericization process and the didactical implications of the analysis.

\section{The idea of generic in mathematics}

In mathematics the same symbol is used to express "for each", "for every" and "for all", namely the universal quantifier " $\forall$ "; this symbol is often employed to convey the idea of generic. We find the fundamental idea of generic already in Euclid (see his generic proof of the infinity of prime numbers) and in some speculations of philosophers (e.g., Locke: see below), but it was particularly exploited within algebraic geometry at the turning of 19th century. The geometers introduced explicitly the notion of generic point, and the related practices in their discipline. However, while generic points were widely used, one cannot easily find their definition. For that, we can refer to Van der Waerden:

Indeed, by generic point of a variety, one usually means, even if this is not always clearly explained, a point which satisfies no special equation, except those equations which are met at every point. (VAN DER WAERDEN, 1926, p. 197; English translation in SCHAPPACHER, 2007, p. 253) 
And to Enriques and Chisini:

The notion of a generic 'point' or 'element' of a variety, i.e., the distinction between properties that pertain in general to the points of a variety and properties that only pertain to exceptional points, now takes on a precise meaning for all algebraic varieties. A property is said to pertain in general to the points of a variety $V_{n}$, of dimension $n$, if the points of $V_{n}$ not satisfying it form - inside $V_{n}-$ a variety of less than $n$ dimensions. (ENRIQUES; CHISINI, 1915, p. 139)

An interesting point of view is given by Speranza (1996), who analysed the idea of "general triangle" through the words of the philosopher Locke: "[The general triangle] must be neither oblique nor rectangle, neither equilateral, equicrural, nor scalenon: but all and none of these at once" (original quotation in SPERANZA, 1996, p. 15). The idea of generic occurs in the mathematical practices leading to the research of a generic stereotype which represents all the basic features desired without any added specific singularity.

In Algebra the generic occurs when we work on generic examples. For instance, let us imagine that we have to decide if the sum of two even numbers is even or odd. In Algebra, we can proceed empirically, testing several cases (e.g., $2+4=6 ; 4+8=12$, and so on) and, then, inducing the general property: the sum is an even number. This is an example of generalization, which means inducing the general case from a sequence of particular cases. From an epistemological point of view, generalization is an empiric induction, which entails an empiric, but not real proof. However, we can follow another way in order to decide if the sum of two even numbers is even or odd: we can reason on a particular example, giving emphasis to a general feature that characterizes all the examples similar to the proposed one.

$$
\begin{aligned}
& 14=7+7 \\
& 22=11+11 \\
& 36=18+18
\end{aligned}
$$

Consequently, it becomes useless to provide other examples: the given one can be conceived as generic. This is a generic proof, that is "a proof carried out on a generic example" (LERON; ZASLAVSKY, 2013, p. 24). From the didactic point of view, the idea of generic example has been interpreted by Mason and Pimm (1984) as seeing the generic in the particular:

A generic example is an actual example, but one presented in such a way as to bring out its intended role as the carrier of the general. This is done by means of stressing and ignoring various key features, of attempting to structure one's perception of it. (MASON; PIMM, 1984, p. 287)

We can find an analogous process in Calculus, when we know how a function behaves for some values of $x$ and we shift our reasoning on a generic abscissa $x$, which must belong to the function domain, but has no particular added characteristics. 


\section{Theoretical framework}

Within the work on functions, the genericization (generating the generic case) is an essential phase in the process of conceptualising the derivative of a function as a function in its turn. According to the structure of a MWS theorised in Kuzniak (2011) and then resumed in Kuzniak and Richard (2014), we focus in particular on the semiotic axis and on process of visualisation (see Fig. 1). This process has to be intended in a large sense, as Kuzniak and Richard point out.

The process of visualization [...] must be associated with the diagrams and the operations of the use of the signs, about which nothing proves a priori that they all pick up the entire visualization as such, even within its extended conceptualization. [...] This process of "extended" visualization has to be well distinguished from the simple vision or perception of the objects; it can be envisaged as a process of structuring the information provided by the diagrams and the signs. It nourishes the intuition of the properties and sometimes contributes to establishing cognitively the validity of these properties. (KUZNIAK; RICHARD, 2014, pp. 20-21)

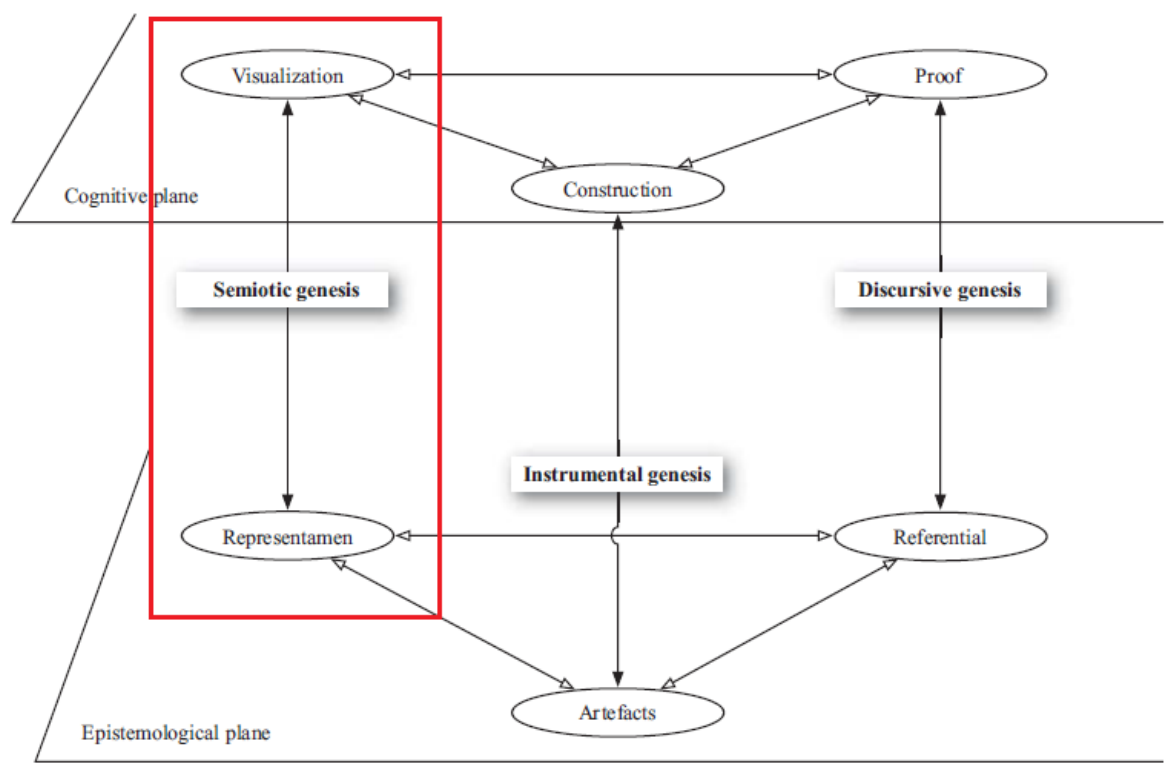

Figure 1 - Semiotic axis within a MWS (KUZNIAK; RICHARD, 2014, p. 21).

A semiotic activity on the involved signs or representamen (in the sense of Peirce ${ }^{1}$ ) leads to define the derivative as a function with its own properties (e.g., domain, sign, asymptotical behaviour, ...) that can be studied in relation to the properties of the primitive functions. In this sense, students get to visualise the derivative function. Reasoning on a prototype by seeing it as a generic example that has all the required properties with no added specificity can be an essential step of the visualisation processes.

\footnotetext{
${ }^{1}$ In a letter to Lady Welby, Peirce wrote: "I define a Sign as anything which is so determined by something else, called its Object, and so determines an effect upon a person, which effect I call its Interpretant, that the latter is thereby mediately determined by the former" (PEIRCE, 1977, pp.80-81; Letter to Lady Welby, 1908).
} 
As one of the facets of the semiotic genesis allowing the back and forth between the epistemological and the cognitive planes, we are interested in the different ways to regard functions while working on them, when different aspects are considered or revealed during the work. They determine different perspectives that it is possible to take on a function.

Following Maschietto (2002, 2008), Rogalski (2008) and Vandebrouck (2011), let us distinguish between pointwise, global and local properties on a given real function $f$ of one real variable. A property of $f$ at a given point $x_{0}$ is pointwise when it depends only on the value assumed by $f$ at $x_{0}$. For instance, $f\left(x_{0}\right)=3$ is a pointwise property which tells us nothing about $f\left(x_{1}\right)$ if $x_{1} \neq x_{0}$. Further, we have global properties of $f$, which are valid on intervals: parity, periodicity, sign, variation, etc. For instance, $f$ is increasing in the interval $(a ; b)$ is a global property. Finally, a property of $f$ is local at the point $x_{0}$ if it depends on the values of $f$ in any neighbourhood of $x_{0}$. For instance, $f$ has limit $l$ in $x_{0}, f$ is continuous or differentiable in $x_{0}, f$ is negligible with respect to another function in a neighbourhood of $x_{0}$, etc. We identify a pointwise perspective when the work on a function is centred on a specific point or a finite set of points. We recognise a global perspective when the interest is on a specific interval or on the whole domain of the function (even when the domain is not explicit). Finally, we detect a local perspective when the focus is on "what the function does" in a neighbourhood of the point, without any specification of the extremes of the considered interval. For instance, by observing that $f$ has a corner point in $x_{0}=1$, we are not able to fix any particular interval containing 1 , but an infinite family of intervals (e.g., $(1-\delta ; 1+\delta)$ ), more or less narrow, can satisfy the (local) property.

Moreover, Vandebrouck (2011) considers the extension of pointwise to universal pointwise properties, which are pointwise properties verified for all the values $x$ in the domain or in a given interval. For instance,

$f$ is even if and only if its definition domain $D$ is symmetric with respect to zero and $\forall x \in D f(-x)=f(x)$.

Notice that every universal pointwise property is expressed through a universal quantifier on the domain or a subset of it. Therefore, a universal pointwise property is a global property, and when we want to verify a global property for the function $f$ on a certain interval $I$ we can also be interested in what $f$ does for each point (i.e., point by point, for every $x$ ) belonging to $I$, instead of looking at the function as a whole over $I$. Thus, we also speak of global perspective achieved as a universal pointwise perspective, when the interest is on the 
values of $f$ point by point and when a pointwise property is considered for a generic value of $x$ belonging to an interval or to the domain (universal pointwise perspective).

Identifying a perspective entails, first of all, clarifying on which object the perspective is activated. Indeed, it has to be pointed out that a certain perspective is always activated with respect to a particular function. To make an example, the claim ' $f$ ' $(2)=3$ " may be read as a pointwise information about $f^{\prime}$ in $x=2$, but also as a local property about the slope of $f$ in $x=2$. In addition, this example shows us that it is certainly necessary to identify that a certain property of $f$ or $f^{\prime}$ is claimed, but also it is essential to analyse the way in which such a claim is made. Sometimes, indeed, we grasp the actual perspective from which a certain proposition is made only if we observe carefully how it is formulated, i.e. how it is semiotically expressed. For instance, some local properties such as " $f$ is discontinuous in $x_{0}$ " often reveals the pointwise corresponding image that the function has a hole in $x_{0}$. On the contrary, sometimes a perspective is not formulated in an uttered claim, but it is implicitly conveyed by a drawing or a gesture. For example, if one claims " $f$ ' $(2)=0$ " we are led to suppose that he is saying something pointwise on $f^{\prime}$. However, if at the same time he moves his hand horizontally on the graph of $f$, we can interpret his claim as a local consideration on $f$.

For this purpose, in our analysis, we use the lens of the semiotic bundle, which has been introduced by Arzarello (2006) to study the relationships among the semiotic resources that are involved in a mathematical activity. He defines a semiotic set as composed of three elements:

- a set of signs (in the sense of Peirce, see Note 1) produced with different intentional actions;

- a set of modes for producing and transforming signs;

- a set of relationships among signs and respective meanings.

A semiotic bundle, in turn, is made up of a collection of semiotic sets and a set of relationships among them. This definition includes the registers of representation as introduced by Duval (1993) but permits also to encompass a variety of semiotic resources used by students and teachers, such as gestures, glances, drawings and extra-linguistic modes of expression. Moreover, the lens of the semiotic bundle allows the study of the relationships within and between the registers simultaneously active and their dynamics, since their activation is multimodal. For instance, according to Arzarello, "speech, gestures and written representations (from sketches and diagrams to mathematical symbols)" are examples of "three different types of semiotic sets" and all together, with the relationships among them, 
"constitute a semiotic bundle, which dynamically evolves in time" (ARZARELLO, 2006, p. 284). Concerning mathematics learning, he proposes two different kinds of analysis:

The first one is synchronic analysis, which studies relationships among different semiotic sets activated simultaneously by the subject. The second is diachronic analysis, which studies the relationships among semiotic sets activated by the subject in successive moments. (ARZARELLO, 2006, p. 287)

Both the model of the mathematical working space (MWS) and the lens of the semiotic bundle refer to Peirce's theory of signs, allowing the work on a particular mathematical object (in our case, the functions). The mathematical work is developed around these signs thanks to an epistemological-cognitive dialectic. Within the model of the MWS, we will use the semiotic bundle as a lens to analyse the semiotic genesis in the interplay between the epistemological and the cognitive dimensions of the work. As a matter of fact, when a subject acts on signs, he/she enacts certain modes for producing and transforming them, creating relationships among them. As a result, he/she comes to activate different semiotic sets (within the semiotic bundle) that enable him/her to recognise certain properties of the mathematical object at stake and so to visualise it (in the sense of Kuzniak and Richard). Therefore, recognising the semiotic resources activated and coordinated within the mathematical work on this object allows us to describe and interpret the entire semiotic activity. The lens of the semiotic bundle indeed, provides us with theoretical tools for studying the semiotic genesis from the activated signs to the visualisation of the object, and vice versa. Actually, especially in the case of functions, visualising a function leads to activate new signs, and working on them adds something to the process of visualisation.

In the case of functions, different semiotic resources can be exploited: speech, gestures and written speech, symbols, sketches or drawings. Their activation and coordination can reveal or hide a particular perspective on the involved functions. When two or more semiotic resources converge to underline the same perspective on a function, this unity may enhance such a perspective and foster its adoption. However, it is also possible that two or more different semiotic resources simultaneously active highlight different perspectives on a function. For instance, it may happen that while uttering the local claim " $f$ is discontinuous in $x_{0}$ ", a teacher or a student makes a pointing gesture on the graph of $f$ in the point $x_{0}$. In this case, the semiotic bundle composed of speech, graph and gesture contains conflicting features regarding the adopted perspective. Such conflicting features may interfere with the intention of conveying a local perspective on the function. 
Concerning this relation between semiotic resources and perspectives, we want to remark that sometimes some semiotic bundles are suitable, more than others, to disclose a certain perspective on functions. For example, accompanying global remarks on a function with its graph may enhance the global perspective on it. Furthermore, recalling the categorization of gestures in McNeill (1992), a pointing gesture might convey a pointwise perspective, whereas a continuous iconic gesture, for example along the graph, may prompt to a global perspective on it. For this purpose, two points appear very useful to detect and to discuss within the analysis:

- which semiotic resources are combined together to convey a specific perspective;

- the concordance/discordance of the perspectives conveyed by the different resources composing the semiotic bundle.

\section{The introduction of the derivative function in the reference MWS}

In this paper, we focus on the introduction of the derivative function at the last year of scientific high school. We will consider how the (Italian) school institution proposes to approach it in the reference MWS and then we will analyse how it is converted by two teachers in an adequate MWS to implement it in their classroom, according to their personal MWS.

From an institutional point of view, there is no explicit reference to the definition of derivative function in the Italian national curriculum. In the mathematics textbooks, the topic is present and it is approached both algebraically and graphically. Nevertheless, in order to solve the problems proposed in the national final examination, students are expected to have conceptualised it as a function, with its own properties in relation with the properties of the primitive functions. See for example the text (Table 1) of one of the problems proposed in June 2013 and the first question ${ }^{2}$.

Hence, we can realise how much the derivative function is a sensitive topic for teachers, who have to prepare their grade 13 students for the final examination without an appropriate curricular support.

\footnotetext{
${ }^{2}$ Source: Ministero dell'Istruzione, dell'Università e della Ricerca, Y557 - Esame di Stato di Liceo Scientifico, corso sperimentale, giugno 2013. Indirizzo: Piano Nazionale Informatica, Tema di: Matematica. Our translation in English.
} 
Table 1 - Excerpt of the text of the Problem 1, given at the final examination (MIUR, June 2013).

A function $\mathrm{f}(\mathrm{x})$ is defined and differentiable, along with
its derivatives of first and second order, in $[0 ;+\infty[$ and
the figure shows the graphs $\Gamma$ and $\Lambda$ respectively of $\mathrm{f}(\mathrm{x})$
and its second derivative $\mathrm{f}^{\prime}$ ' $(\mathrm{x})$. The tangent to $\Gamma$ in its
inflection point, whose coordinates are $(2 ; 4)$, passes
through $(0 ; 0)$, while the straight lines $\mathrm{y}=8$ and $\mathrm{y}=0$
are horizontal asymptotes respectively for $\Gamma$ and $\Lambda$.
Prove that the function $\mathrm{f}^{\prime}(\mathrm{x})$, that is the first
derivative of $\mathrm{f}(\mathrm{x})$, has a maximum and determine
its coordinates. Knowing that for each $\mathrm{x}$ in the
domain it is $\mathrm{f}^{\prime}$ ' $(\mathrm{x}) \leq \mathrm{f}^{\prime}(\mathrm{x}) \leq \mathrm{f}(\mathrm{x})$, what is the
possible graph of $\mathrm{f}^{\prime}(\mathrm{x})$ ?

Getting to the representation of the derivative function, in the algebraic register, entails a semiotic activity on the signs $x_{0}, f^{\prime}\left(x_{0}\right), \lim , h \rightarrow 0$ and the algebraic expression of the incremental ratio of the function $f$ in the intervals $\left(x_{0} \pm h ; x_{0}\right)$. Starting from the derivative of the function $f$ defined at the point $x_{0}$ as $f^{\prime}\left(x_{0}\right)=\lim _{h \rightarrow 0} \frac{f\left(x_{0}+h\right)-f\left(x_{0}\right)}{h}$, one of the most widespread textbooks in Piedmonts' schools writes

We can calculate the derivative of a function also at a generic point. In this case, the obtained value $f^{\prime}(x)$ is a function of $x$ and, for this reason, we speak also of derivative function. [...] The derivative function, as $x$ varies, provides the gradient of all the tangent lines to the given function. (BERGAMINI et al., 2013, p. 1621; our underlining)

Some lines after, in a guided exercise, we can read the symbolic formula $f^{\prime}(x)=\lim _{h \rightarrow 0} \frac{f(x+h)-f(x)}{h}$.

The semiotic technique consists in substituting $x_{0}$ with $x$ and it is grounded on the idea of considering a generic point $x$. Behind this technique, there is a process of genericization through which $x_{0}$ is seen as a generic abscissa $x$ and loses any link with a specific real number belonging to the function domain. The examined textbook does not deepen any further justification of this technique. Hence, our research focus shifts on teachers: how do they manage this process with their students?

\section{Methodology for teachers' practices observation}

Three mathematics teachers have joined up our research project. In this paper, we are going to present the cases of M. and V., experienced teachers in two different scientific high 
schools in Piedmont (Italy). For both teachers, it was the third year with the same students: they taught them also in grade 11 and 12. At the beginning of the study (September 2012), they used one of the two mathematics textbooks for grade 13 classrooms that we had consulted, and they were preparing their students for the national final examination that we discussed above.

We revealed to the two teachers the basic intention of our research project, namely: to study the teaching practices related to the derivative concept. Initially, we told them nothing about our analysis lenses, so that the lessons could follow their natural course. The context was necessarily an unusual one, due to the presence of an external observer. Our concern was to influence the regular lesson development no more than that.

The data collection consists of three phases. We preliminarily interviewed the teachers about their usual practices with the derivative and their planning for teaching it during that year. We observed each teacher's lessons, especially those devoted to the introduction of the derivative notion and the definition of the derivative function. We had intermediary informal meetings with teachers where we could ask them reasons for some decisions. Finally, we proposed two activities to the students: two sets of problems properly designed on the derivative, to be solved in homogeneous groups.

In this paper, we specifically analyse some video excerpts of the teachers' lessons, coming from the observation in classroom at the moment of introduction of the derivative function in the algebraic register of representation.

\section{Analysis of teachers' practices with the idea of generic}

According to our theoretical framework, studying how the teacher manages the process of genericization with her students entails the analysis of the adequate MWS implemented by the teacher in the classroom, according to her personal MWS. A special focus is on the dialectic of perspectives on functions that accompanies the shift from the pointwise definition of the derivative of a function at a point $x_{0}$ to the global definition of the derivative function. Moreover, we analyse which semiotic resources are activated and how they are combined in order to foster the shift in perspectives.

\subsection{The case of $M$.}


M. is giving her first lesson on the derivative notion to her grade 13 students. We are going to analyse an excerpt of about thirty minutes from this first lesson. To give some contextual elements, M. has just introduced the derivative of a function $f$ at a point $x_{0}$ as the limit of the incremental ratio of $f$ as the increment $h$ goes to 0 (Fig. 2). The derivative has been defined as the gradient of the tangent line to $f$ at $x_{0}$ seen as the limit of a sequence of secant lines.

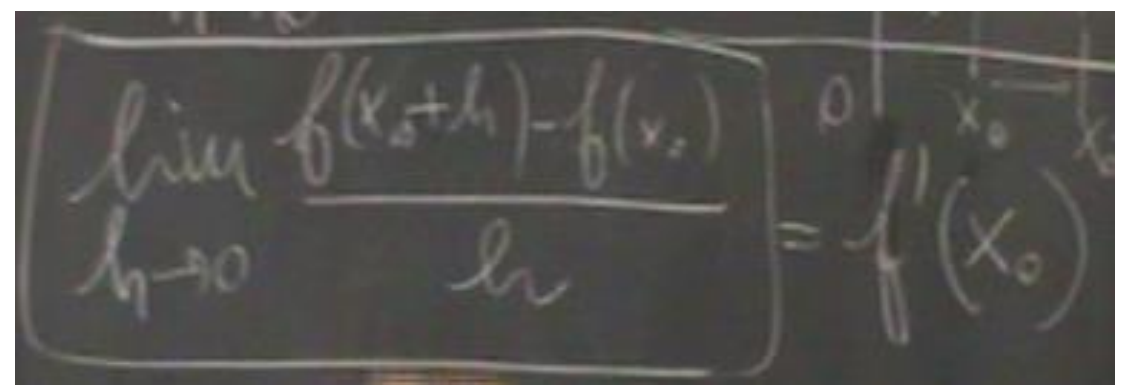

Figure 2 - M.'s technique for the derivative of a function $f$ at a point $x_{0}$.

M. proposes to work on an example, in order to practice the new technique. She gives and solves the following task: determining the derivative of the function $y=x^{2}$ in the point of abscissa $x_{0}=2$. She uses the definition in Fig. 1 and finally finds $f^{\prime}(2)=4$. Notice that, in the teacher's intentions, the given task and the technique used for solving it are pointwise on the function $f^{\prime}$. Let us analyse how she comments the exercise and introduces the following one (in the transcript, Sn stands for the student $\mathrm{n}$ ).

1 M: Now, to summarise, what have we done? The concept of derivative, but calculated at a point. (She points to an imaginary point in front of her, with her left hand, Fig. 3) [...] The derivative of a function at a point, what does it give?

2 S1: A coefficient.

$3 \quad$ M: A number, exactly. But now let's make a step forward. We have $y=x^{2}$ and we have calculated the gradient of the tangent line in the point $x_{0}=2$. (She repeats the same previous gesture with her left hand, as in Fig. 3) If now I ask you "What is the value of the gradient at the point with abscissa $x_{0}=5$ ?" One should again work hard and do all the calculation. Right? At $x_{0}=1 \ldots$ and so on. (She moves her hands as if something is rolling in front of her) You see, it's not so convenient, also from a practical point of view.

$4 \quad$ M: $\quad$ So, what shall I do? The calculation at a generic point $x$. (She joins upwards the fingers of her right hand and then turns them down on the left open palm, Fig. 4) Ok? That is, instead of calling it $x_{0}$, I call it $x$. (She repeats the same gesture as before, Fig. 4)

$5 \quad$ M: $\quad$ And now we must be really careful! I call it $x$. Which outcome do I expect?

$6 \quad$ S2: A function.

$7 \quad$ M: $\quad$ Can it be a number?

8 S3: With $x$.

$9 \quad$ M: Yes, it will be a function of $x$. So, you understand that we can speak about "derivative function", which will be again a function of $x$.

$10 \quad$ S4: And then we can replace inside it...

$11 \quad$ M: $\quad$ Perfect! S4 is saying "Of course, then, if I want the gradient of the tangent in the point $x_{0}=5$, it will be sufficient to put $x=5$ in the derivative function". Let's do it! 

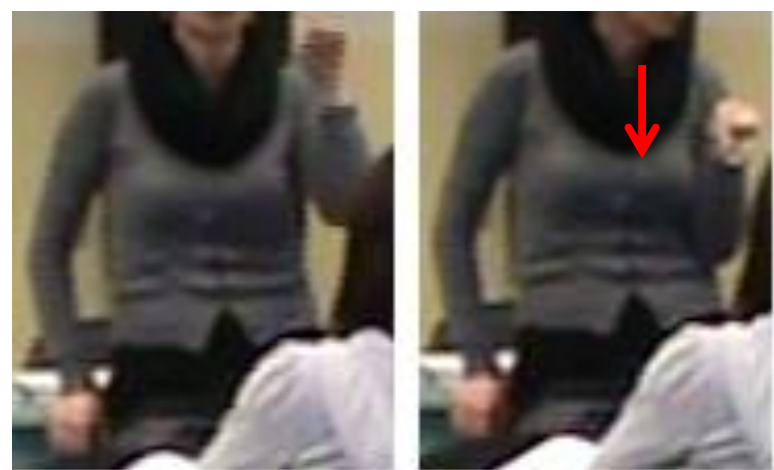

Figure 3 - M.'s gesture for "the point $x_{0}$ " [lines 1, 3].
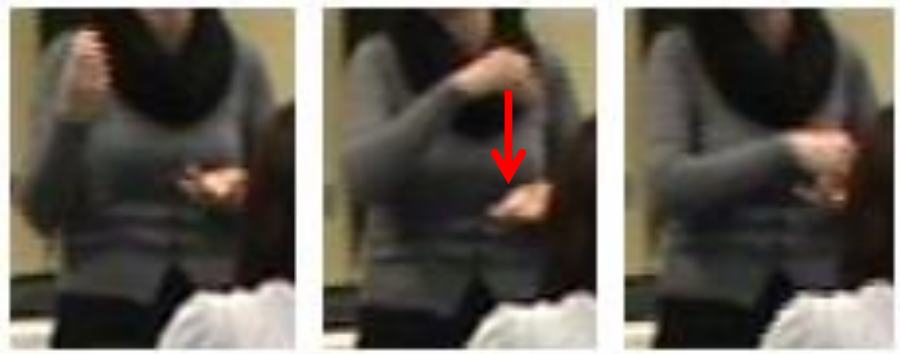

Figure 4 - M.'s gesture for the "generic point $x$ " [line 4].

After this comment, the teacher solves the same task following the same steps with $x$ instead of $x_{0}$. She obtains $f^{\prime}(x)=2 x$.

The first utterance and the first gesture used by M. [line 1] stress that the starting perspective is pointwise on $f$ '. Then, she underlines that making other numerical examples is actually useless, since every further example (with $x_{0}=5$, with $x_{0}=1$, etc.) would always entail the same calculations done for $x_{0}=2$ [line 3]. The case of $x_{0}=2$ is becoming a generic example. M. uses the semiotic technique of replacing $x_{0}$ with $x$ [line 4], where the generic sign $x$ represents "every value of $x_{0}$ ". Even M.'s gesture for accompanying the universal pointwise expression "the generic point $x$ " [line 4] is different from the one used before for referring to "the point $x_{0}$ " [line 1] (see Figures 3 and 4). In the adequate MWS that M. implements in her classroom, the shift from $x_{0}$ to $x$ is introduced after the limit calculation done for a specific value of $x_{0}\left(x_{0}=2\right)$. This choice is important within the mathematical work that $\mathrm{M}$. considers as adequate for making her student visualise the derivative as a function. Indeed, her argument is based on the fact that using a generic $x$ avoids them to repeat many times the same and long limit calculation [line 3]. We can find confirmation about the importance of justifying the used semiotic technique through this particular choice during one of intermediary meetings with M. Her words highlight that it depends on her personal MWS:

Interviewer: Can you tell me something about the choices you made about the introduction of the derivative function: moment of the lesson, methods, ...?

M: I always think about it: starting from the derivative function at a point, which is a number, one must obtain the function. This is really a delicate step. It is a sort of conquest: every time, 
I make a calculation that I could actually make only once. I find that if you [teacher] give to students a justification of what you are doing, they get it better.

Finally, the teacher makes the students reflect upon the global expectations on the result [lines 5-9]: $f^{\prime}(x)$ is expected to be globally a function of $x$. Asking "Which outcome do I expect?" [line 5] M. wants the students to reflect on the status of the obtained sign (i.e., $f^{\prime}(x)$ ) and of its algebraic expression that will depend on the generic $x$ that they have just introduced. Within M.'s adequate MWS, this reflection can be seen as a way of fostering students' visualisation of the derivative as a function.

Moreover, the student S4 remarks that we can replace $x$ in $f^{\prime}(x)$ with any number we wish [lines 10-11]. This is a further technique for finding out the derivative of $f$ at a particular abscissa $x_{0}$ : finding the algebraic expression of $f^{\prime}(x)$ and then replacing $x$ with $x_{0}$. Such a technique is supported by the whole speech that M. has just done in the classroom around the signs $x_{0}$ and $x$ and $f^{\prime}$ as a function of $x$. S4's proposal shows that he is visualising the derivative as a function, since he is able to coordinate the global perspective (at least in the sense of universal pointwise) and the pointwise perspective on it.

Notice that M. grounded the shift from the pointwise to the global perspective on $f$ ' on the syntactical process, which is typical of the algebraic symbolic writing: from a particular $x_{0}$ to a generic $x$. This is the classical algebraic technique of the replacement of a variable with its value and vice versa.

\subsection{The case of $V$.}

During the first lesson on the derivative notion, V. and her students have found together the formula of the derivative of a function $f$ at a point $x_{0}$ (see Fig. 5), as the gradient of the tangent line to $f$ at $x_{0}$. They have started from the students' definition of tangent line as the best linear approximation of the function in a neighbourhood of $x_{0}$. The result is the technique in Fig. 5 (on the left), that is the limit of the incremental ratio of $f$ as $x$ goes to $x_{0}$. We are going to analyse an excerpt of about thirty minutes from the third lesson, which V. starts by showing the equivalent technique for $h$ that goes to zero (Fig. 5 on the right).

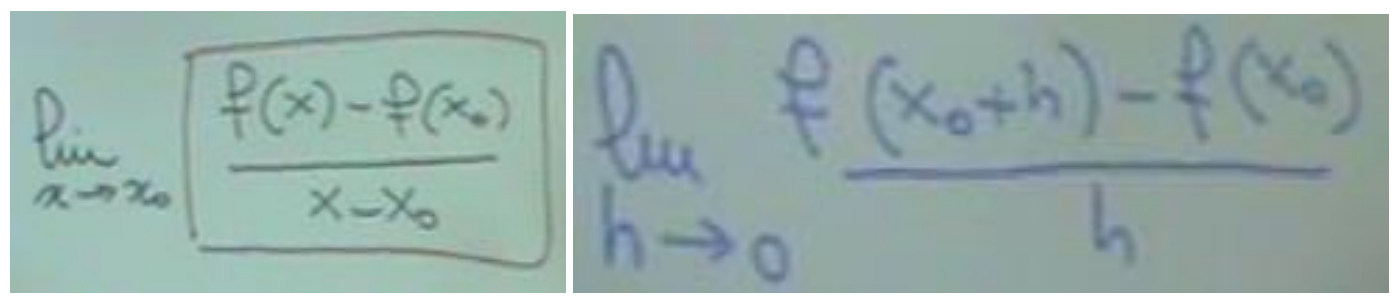

Figure 5 - V.'s techniques for the derivative of a function $f$ at a point $x_{0}$. 
Afterwards, V. gives a task to the students:

$1 \quad V: \quad$ We are going to calculate the limit of the incremental ratio for the function $f(x)$ $=x^{2}$. Try to write it on your own. So, try to calculate the derivative for $x^{2}$ in any point $x_{0}$ of it.

2 S1: Any point?

$3 \quad$ V: $\quad$ Any point. As usual, let's call it $x_{0}$.

Notice that, according to the teacher's goal of introducing the derivative function, the given task aims at triggering a universal pointwise on $f^{\prime}$ ["any point $x_{0}$ ", line 1] and the techniques the students dispose of are pointwise on f' (Fig. 5). V.'s utterance in line 3 seems to reveal that the class is somehow familiar with the work on generic signs.

The students work alone for a while, the teacher walks through the classroom. When a part of the class has solved the task, she makes all the steps at the whiteboard, obtaining $2 x_{0}$. Then, she comments as follows:

$4 \quad V: \quad$ What have I discovered? I've discovered that when I have the function $x^{2}$, its derivative is, point by point, is $2 x_{0}$.

$5 \quad V: \quad$ So, if I write a function here, and its derivative here (she starts composing a table $f \mid f$ ' at the whiteboard), I've discovered that the derivative of the function $x^{2}$ is $2 x$. (She writes " $x^{2} \mid 2 x$ " in the first row of the table $f \mid f$ ") This is an automatic process, because if I have $x^{2}$, from this moment on, I won't calculate the limit of the incremental ratio anymore. I know that its derivative is $2 x$. I've calculated it once and for all, in the general case of any point $x_{0}$, so I have it.

When V. finds the result $2 x_{0}$, she globally interprets it as "the derivative point by point" [line 4], which actually recalls the universal pointwise perspective on $f^{\prime}$ of the given task [lines 1-3]. V. suddenly replaces $x_{0}$ with the global variable $x$ [line 5]. This semiotic technique is implicit in the change of signs from line 4 to line 5 . In the adequate MWS that V. implements in her classroom, the shift to a generic value $x_{0}$ is proposed before the limit calculation. This choice is important within the mathematical work that V. considers as adequate for making her student visualise the derivative as a function. Indeed, after the limit calculation in $x_{0}, \mathrm{~V}$. bases the drastic change of $x_{0}$ into the $x$ variable on the fact that the considered $x_{0}$ was generic [line 5]. The teacher uses the table $f \mid f^{\prime}$ as a resource for structuring the findings in a schematic way. As for the perspectives, $x_{0}$ is used as a universal pointwise sign, representing every abscissa $x_{0}$ of the domain, while $x$ has the global meaning of variable. At this stage V. does not make explicit the shift from $x_{0}$ to $x$. It follows a technique that is not clear for students. A student intervenes about the change of signs.

$6 \quad$ S2: $\quad$ The independent variable changes from f to $f^{\prime}$. Is it $x_{0}$ or is it always the same?

$7 \quad V: \quad$ It is a point $x$. [...] Let's take $f(x)=x^{2}$, which I'm able to draw, that is the parabola (she draws the curve). What have we discovered and proved? That if I take any point $x_{0}$ (she chooses a point $x_{0}$ on the $x$-axis), then the gradient of the tangent line at the point of abscissa $x_{0}[\ldots]$ is $2 x_{0}$. So, if I draw the tangent line here (she traces the tangent at the 
correspondent point on the parabola), this straight line has $2 x_{0}$ as gradient (she writes $m=$ $\left.2 x_{0}\right)$.

$8 \quad V: \quad$ What does it mean? It means that I can make $x_{0}$ vary as I want (she moves her hand forwards and backwards, Fig. 6). At this point, I can write $x$ instead of $x_{0}$, for convenience.

$9 \quad V: \quad$ And point by point I have a formula, that is the following (she writes $f^{\prime}(x)=$ $2 x$ ) which point by point (she moves the stick as in Fig. 7) tells me the value of the gradient of the tangent line.

$10 \quad S 2: \quad[\ldots] f^{\prime}(x)$ gives me the gradient.

$11 \quad V: \quad Y e s$, as $x$ varies. So, the variable is the same. Point by point, here I have a function that point by point automatically, as a machine, tells me the gradient of the tangent line.

12 S2: Only, I don't understand the passage. If we know that $m$ is $2 x, f(x)$ corresponds to $y$, while $m$ corresponds to the tangent. How can they be equivalent? I don't understand.

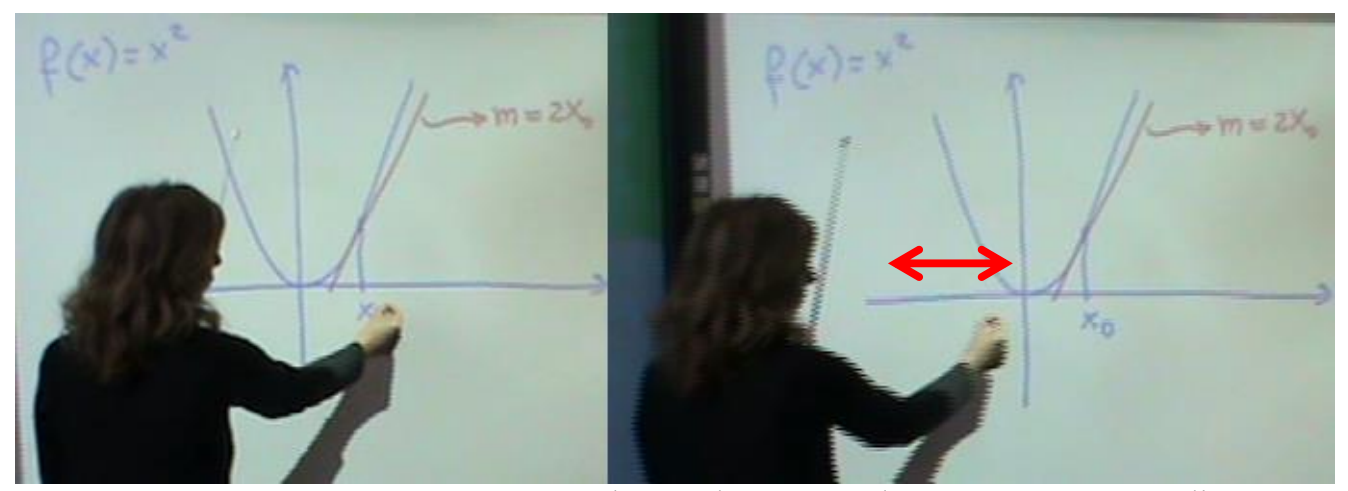

Figure 6 - V.'s gesture to accompany the words "I can make $x_{0}$ vary as I want" [line 8].

As we can infer from S2's intervention [line 6], the semiotic technique introduced by V. induces doubts for the students about the variables at stake. The teacher clarifies the generic role of $x_{0}$ and justifies the shift from $x_{0}$ to $x$, which was not so explicit in lines 4-5. V. starts from stressing the pointwise basic character of the sign $x_{0}$, by choosing a particular point on the $x$-axis and the corresponding one on the parabola $y=x^{2}$. In line 7 , we can notice an incongruity that typically occurs when one uses the graphical resource to speak of something generic. One declares to consider a generic point on the curve, any value of the abscissa $x_{0}$. However, when detected on the drawing, the point or the abscissa necessarily becomes a specific point on the curve or a specific value of the abscissa. In order to regain generality and variability, V. uses the speech "I can make $x_{0}$ vary as I want [...] I can write $x$ instead of $x_{0}$ " [line 8] and continuous gestures on the graph (see Fig. 6). The previous hint to the technique [lines 4-5] is a little bit developed here. She justifies the change of $x_{0}$ into $x$ as a convenience. Actually, she is giving to the generic universal pointwise sign $x_{0}$ the global status of variable. It occurs through the combination of V.'s utterance [line 8] and her continuous gesture (Fig. 6) on the graph. As a consequence, the perspective on the functions $f(x)$ and $f^{\prime}(x)$ would be global in the sense of variable. Nevertheless, when the teacher makes 
explicit the global perspective on $f^{\prime}(x)$, she enhances the universal pointwise character of the formula $f^{\prime}(x)=2 x$, which "point by point tells me the value of the gradient of the tangent line" [line 9]. Instead, her gestures on the function $f$ (see Fig. 7) are continuous and global.

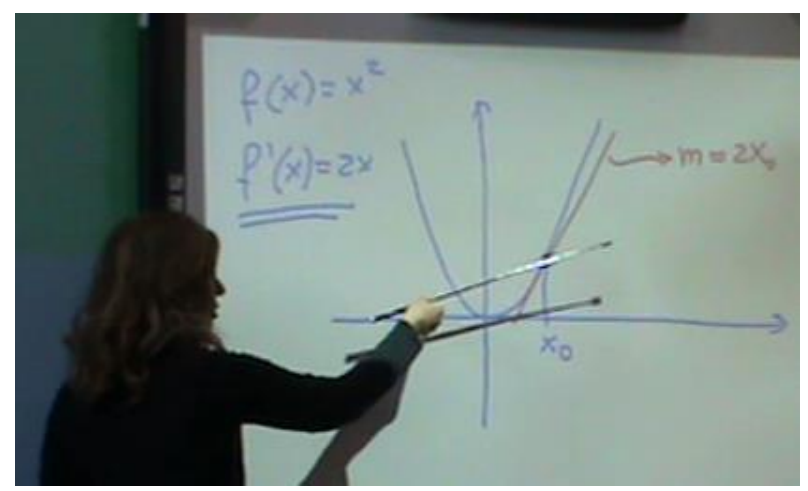

Figure 7 - V.'s gesture to show the tangent "point by point" [line 9].

In V.'s words [line 11] we find the definition of the derivative $f$ ' as "a function that point by point automatically, as a machine, tells me the gradient of the tangent line". The explicit perspective on $f^{\prime}$ is global, achieved as universal pointwise. However, the new doubt of the student $\mathrm{S} 2$ is about the status of $f^{\prime}$ as a function [line 12]. He has a clear global perspective on the function $f$, thanks to the graphical register used in the teacher's drawing, but he cannot understand how also the gradient $m$ (and so $f^{\prime}$ ) could behave as the function $f$ does. From his words, we can infer that the confusion on the used signs, at this stage, represents an obstacle for the student's visualisation of the derivative as a function.

\section{Discussion}

The aim of this paper is to investigate how teachers manage the process of genericization with their students at the moment of introducing the derivative function. We have reported on the analysis of the practices of two Italian teachers, V. and M.. In both cases, the teachers lean on the algebraic register of representation, starting from the definition of the derivative at a specific point $x_{0}$. In several Italian textbooks (e.g., BERGAMINI et al., 2013), the given technique consists in the syntactical change of the pointwise sign $x_{0}$ into the global sign $x$ (see §4). In the teachers' words, this technique is expressed through the words "Instead of calling it $x_{0}$, I call it $x$ " (see M., line 4) or "I can make $x_{0}$ vary as I want [...] I can write $x$ " (see V., line 8). Both the teachers ground the justification of the used semiotic technique on the idea of making $x_{0}$ generic and so of considering a generic $x$. In terms of adequate MWS, both teachers start from the task of calculating the derivative of the function $f(x)=x^{2}$ at $x_{0}$. In a case, M. makes the numerical example with $x_{0}=2$ become generic and reformulates the 
starting pointwise task in terms of a generic abscissa $x$. In the other case, V. immediately formulates the task in terms of a generic $x_{0}$ and then gives to it the global sense of the $x$ variable. M. introduces the shift from a specific $x_{0}$ to the generic $x$ after the calculation of the limit, whereas V. speaks of a generic sign $x_{0}$ before the limit calculation. Although based on the same semiotic technique of replacing $x_{0}$ with $x$, the adequate MWSs turn out to be different. This difference in the teacher's choice is likely to influence the way the students understand the implications of replacing $x_{0}$ with $x$ in the involved functions and the justification behind this semiotic technique. We can suppose that doing the limit calculation in $x_{0}$ (even if it is "any $x_{0}$ ") the students are more inclined to imagine a pointwise result, whereas doing the limit calculation in $x$, the expectation that the result is a global function of $x$ might be stronger. A deeper analysis of the students' work and their personal MWS would be necessary to verify this hypothesis.

The process of genericization occurs at the level of the independent variables $x_{0}$ and $x$, but it influences the mathematical work on the dependent variable expressed by $f^{\prime}(x)$ in terms of the activated perspectives. In the two analysed examples, a shift of perspectives on the derivative function corresponds to the genericization from $x_{0}$ to $x$ : from pointwise to universal pointwise. While on the function $f$ the global perspective can be reinforced, as in the case of V., through the coordination of graph and continuous gestures on it, on the derivative function at this stage the employed semiotic resources are mainly symbols and speech, which are used in a universal pointwise perspective (e.g., "any point", "generic point”, "point by point", ...). The definition of $f^{\prime}(x)$ as a function is formulated verbally: "a function that point by point automatically, as a machine, tells me the angular coefficient of the tangent line" (see V., line 11). Notice that this is a global definition achieved in a universal pointwise perspective. At a cognitive level, it may not be sufficient to get to conceptualise the derivative as a global function. The observed teachers propose to construct the graph of the derivative some sessions after. Thanks to the work on the derivative graph, the global perspective on the derivative function might be enhanced, but it is necessary to establish carefully the relationships between the graph and the semiotic resources (i.e., symbols and speech) through which the derivative function has been introduced, strengthening the meaning of the used signs and variables.

Within both the adequate MWSs of functions, the genericization on the independent variable allows the access to the "visualisation" of the derivative function. Our findings suggest that it is essential to continue fostering and enriching students' visualisation of this 
mathematical object, so that they can be able to work on it as a function and to achieve its properties. From our analysis, a first development of the work on the derivative function seems to make evolve the global perspective, grasped in a universal pointwise way. For instance, it is important to make explicit the character of variable that $x$ has, for shifting from a point-to-point vision to a global vision on intervals. In this process, the coordination of different semiotic resources appears essential, and in particular the use of the graph accompanied by speech and gestures. The semiotic bundle graph-gesture-speech used by V. on the function $f$ (see Fig. 6-7) highlights the global character of $f$ as the variable $x$ varies. A similar work on the derivative function could be planned successfully.

\section{Conclusion and implications on teaching}

From discussing our case studies, one first remark turns out to be relevant in teachers' Calculus practices. While the teacher structures the adequate MWS of functions in her classroom, she has to be very careful with regard to the perspectives activated on the involved functions. The introduction of the derivative function has been presented as a significant example where the dialectic of perspectives on functions must be carefully managed to be a resource, and not an obstacle, for students' visualisation of the derivative as a function. Highlighting universal pointwise properties of $f^{\prime}$ in order to make the pointwise perspective evolve is a useful strategy. However, this may not be enough, at least not for all the students, for constructing a full global perspective on the derivative function.

Analysing teachers' discourses, Park (2015) highlights "specific disconnects between, on the one hand, mathematical concepts and, on the other hand, the words, symbols, graphs and gestures used to communicate them" (PARK, 2015, p. 249), and recommends to reduce the implicitness of some aspects of the derivative. In addition, we recommend a careful choice of the semiotic resources and particular attention to their coordination within the MWS in order to convey a certain perspective. Indeed, every semiotic resource, whether the user is conscious or not, has a certain potential with respect to a particular perspective. For example, drawing the graph of a function may foster a global perspective on it, and making a continuous gesture on the graph of a function (like V.'s gestures in Fig. 6 and 7) may inhibit a pointwise perspective on it, in favour of a global one. Nevertheless, using a semiotic resource that fosters a global perspective can help but may not be enough to obtain its activation, at least not for all the students. Therefore, in the work on functions, it is necessary not only a 
proper choice of the used resources, but possibly also the combined activation of other signs in order to focus the students' attention on the desired perspective.

In conclusion, perspectives and semiotic resources activated on functions, with attention to their mutual interactions, seem to be two important points in the teacher's management of the MWS of functions. So far, we have focused specifically on the semiotic axis of the MWS, but we have also evoked the properties of the involved functions that this kind of work permits to recognise and to treat, that is the referential involved. A further study of the possible influences of the other axis and processes could be a future development of this research.

\section{References}

ARZARELLO, F. Semiosis as a multimodal process. Revista latinoamericana de investigación en matemática educativa, Mexico, v. 9, n. 1, p. 267-300, nov. 2006.

BERGAMINI, M.; TRIFONE, A.; BAROZZI, G. Matematica.blu 2.0. Bologna: Zanichelli, 2013. v.5.

DOUADY, R. Jeux de cadres et dialectique outil-objet. Recherches en didactique des mathématiques, Grenoble, v. 7, n. 2, p. 5-31, 1986.

DUVAL, R. Registres de représentation sémiotique et fonctionnement cognitif de la pensée. Annales de didactique et de sciences cognitives, Strasbourg, v. 5, p. 37-65, 1993.

DUVAL, R. A cognitive analysis of problems of comprehension in a learning of mathematics. Educational studies in mathematics, Netherlands, v. 61, n. 1-2, p. 103-131, Feb. 2006.

ELIA, I. et al. Relations between secondary pupils' conceptions about functions and problem solving in different representations. International Journal of Science and Mathematics Education, Dordrecht, v. 5, n. 3, p. 533-556, 2007.

ENRIQUES, F.; CHISINI, O. Lezioni sulla teoria geometrica delle equazioni e delle funzioni algebriche. Bologna: Zanichelli, 1915.

KUZNIAK, A. L'espace de travail mathématique et ses genèses. Annales de didactique et de sciences cognitives, Strasbourg, v. 16, p. 9-24, 2011.

KUZNIAK, A.; RICHARD, P. R. Spaces for Mathematical Work: Viewpoints and perspectives. Revista Latinoamericana de Investigación en Matemática Educativa, Mexico, v. 17, n. 4-I, p. 17-27, nov. 2014.

LERON, U.; ZASLAVSKY, O. Generic proving: Reflections on scope and method. For the Learning of Mathematics, New Brunswick, Canada, v. 33, n. 3, p. 24-30, nov. 2013.

MASCHIETTO, M. L'enseignement de l'analyse au lycée : les débuts du jeu global/local dans l'environnement de calculatrices. 2002. 395. Thesis (Doctorate in Mathematics Education) - Université Paris 7 et Università di Torino, Paris, 2002. 
MASCHIETTO, M. Graphic calculators and micro-straightness: analysis of a didactic engineering.

International Journal of Computers for Mathematical Learning, Netherlands, v. 13, n. 3, p. 207230, dic. 2008.

MASON, J.; PIMM, D. Generic examples: Seeing the general in the particular. Educational Studies in Mathematics, Netherlands, v. 15, n. 3, p. 277-289, aug. 1984.

MIUR, Ministero dell'Istruzione, dell'Università e della Ricerca. Esame di Stato di Liceo Scientifico, corso sperimentale, giugno 2013. Retrieved from

<http://archivio.pubblica.istruzione.it/argomenti/esamedistato/secondo ciclo/prove/2013/as2013.htm>

Access in: 27 oct 2014.

MCNEILL, D. Hand and mind: what gestures reveal about thought. Chicago: University of Chicago Press, 1992.

MONK, G. S. Students' understanding of functions in calculus courses. Humanistic Mathematics Network Journal, v. 9, p. 21-27, feb. 1994.

PANERO, M.; ARZARELLO, F.; SABENA, C. Practices of Italian teachers with the derivative concept: a problematic meeting between Algebra and Analysis in secondary school. In: GÓMEZCHACÓN, $M^{\mathrm{a}}$ I. et al (Ed.). Mathematical working space. Proceedings Fourth ETM Symposium. Madrid: Publicaciones del Instituto de Matemática Interdisciplinar, Universidad Complutense de Madrid, 2015, p. 605-617.

PARK, J. Is the derivative a function? If so, how do students talk about it?. International Journal of Mathematical Education in Science and Technology, v. 44, n. 5, p. 624-640, jul. 2013.

PARK, J. Is the derivative a function? If so, how do we teach it?. Educational Studies in Mathematics, Netherlands, v. 89, n. 2, p. 233-250, may. 2015.

PEIRCE, C. S. Semiotics and significs: the correspondence between Charles S. Peirce and Lady Victoria Welby. Bloomington, IN: Indiana University Press, 1977.

ROGALSKI, M. Les rapports entre local et global: mathématiques, rôle en physique élémentaire, questions didactiques. In: VIENNOT, L. (Ed.). Didactique, épistémologie et histoire des sciences. Paris: Presses Universitaires de France, 2008, p. 61-87.

SCHAPPACHER, N. A historical sketch of BL van der Waerden's work in algebraic geometry: 1926 1946. Episodes in the history of modern algebra (1800-1950), Providence, RI: American Mathematical Society, 2007, p. 245-283.

SIERPINSKA, A. On understanding the notion of function. In: DUBINSKY, E., HAREL, G. (Ed.), The concept of function: aspects on epistemology and pedagogy. Washington DC: Mathematical Association of America, 1992, p. 25-58.

SPERANZA, F. "Il triangolo qualunque" è un qualunque triangolo?. L'Educazione Matematica, Cagliari, v. 17, p. 13-27, 1996.

VANDEBROUCK, F. Perspectives et domaines de travail pour l'étude des fonctions. Annales de Didactiques et de Sciences Cognitives, Strasbourg, v. 16, p. 149-185, 2011.

VAN DER WAERDEN, B. L. Zur Nullstellentheorie der Polynomideale. Mathematische Annalen, città, v. 96, n. 2, p. 183-208, 1926. 
VINNER, S. Concept definition, concept image and the notion of function. The International Journal of Mathematical Education in Science and Technology, v. 14, n. 3, p. 293-305, jul. 1983.

Submetido em Julho de 2015. Aprovado em Setembro de 2015. 